\title{
Impressions d'Elvira
}

\author{
Claudine Potvin
}

\section{Impressions of Elvira}

Elvira had watched the birth of Julienne fallen crazily in love the spectacle of a twin sister not identical similar... Julienne had seen Elvira move out had followed nothing left to stick around for... at the age of five already taken along to her sister's photographic sessions, her poses, she said, installing her in a chair at the end of a rough room and then making her way over to that ridiculous bed the camera lowered down on sweating skin tangled bodies of strangers loss of equilibrium vanishing species a little girl no longer looking through the keyhole numb in the middle of the scene... by and by Elvira matured under the eyes of Etienne the photographer earning his living on the backs of women she gave her very best went home to her little sister gave her a good hot bath rubbed her back played with her hair fed her put her to bed side by side they dreamed together of horses of woods of bodies smoother than snow but never of the sea... what can happen between two sisters linked by such an unbreakable bond? start the simulacrum over again, backwards, protect the little one, refuse her the right to grow up or remove the big sister from that allegorical chariot heaped with vulvas tableau of breasts mismatched buttocks... something always slips away from mothers that passage from tenderness to indifference a crumb lost in the urban forest an arm a leg a mauve eye some hairs the back of a man a monkey's tail red mouths Elvira had never predicted Julienne's escape the photographer had seen it all in advance... Julienne wants to be like one of Gauguin's women all ripe coloured he's dyed her hair a screaming red... Elvira waiting out in the hallway on a ridiculous stool pressure of fingernails in her palm the smell of blood choking back tears all those penises swallowed like a row of soldiers now caught in the back of the throat all of that only to have produced a twin, they were almost identical weren't they... Julienne henceforth contained in that avid little mouth an internal sea, a pleasure in being looked at, figuring all of those crawling fingers to herself as a nest of ants, dreaming of her future as a filmmaker... Elvira's heart shattered in a million pieces. She killed 
Etienne with a knife. The little one took off. Later, she was seen strutting around on the corner of Saint Catherine and Papineau. Elvira never gets any visitors. On her cell wall, she's stuck photos of her little sister dressed in a pretty white knit dress.

elles se ressemblaient comme deux gouttes d'eau on aurait dit des jumelles à peine dix ans de différence la plus jeune avait vu la plus vieille s'éloigner de la maison se faire un chemin dans la vie l'avait suivie rien d'autre n'avait d'importance personne d'autre ne méritait que l'on reste

à l'âge de cinq ans déjà l'autre la traînait avec elle pendant les séances de photographie pendant les poses, disait-elle, l'installait sur une chaise au fond d'une chambre rude lui donnait un baiser dans le cou s'enfargeait dans les fils les caméras l'éclairage des deux on aurait dit que c'était elle la plus jeune une vraie tête de linotte

la plus vieille avait vu naître la première s'en était amourachée comme d'une folle le spectacle d'une soeur jumelle non identique semblable

entre les deux s'installa une complicité maintenue à coup de malentendus de promesses d'amour éternel d'une précise observation la plus petite ayant assisté pendant de longues heures au déshabillage progressif de sa grande soeur au coeur rétréci, foulé tel un tricot abîmé déformé une mince feuille de papier sur laquelle elle griffonnait à la mine quelques mots le soir avant de s'endormir

les lieux avaient d'abord surpris la petite peu habituée à l'aveuglement et à l'obscurité et puis ce lit ridicule où s'entassaient les corps étrangers le clic de l'appareil dévalant sur les sexes en sueur comme sur des pistes dangereuses elle-même se sentait perdre l'équilibre espèce en voie de disparition une petite fille regarde non plus par le trou de la serrure au milieu de la scène presque en attente en transit transie sous la salive et sous la morve des autres

la plus vieille avait ainsi continué de grandir dans l'objectif d'un photographe qui gagnait sa vie sur le dos des femmes tentant de les rassurer elles ne travaillaient que quelques heures elle offrait le meilleur d'elle-même revenait fraîche et dispose à la maison avec sa petite soeur lui préparait un bon bain chaud avec de la mousse en masse lui grattait le dos lui jouait dans les cheveux la nourrissait la couchait encore l'endormait s'assoupissait à ses côtés elles rêvaient ensemble de chevaux de bois et de corps plus lisses que la neige mais jamais de mers d'eaux limpides ou de désert la nuit elles se réveillaient l'une l'autre pour 
échanger le dernier conte la dernière heure jusqu'au petit matin quand la brise délicate d'un printemps venu trop tôt leur ramenait la fragilité du jour dans les yeux des filles démaquillées en ce temps-là elles n'en finissaient plus de se reposer remettant la même robe pour ne pas la laver taisant la peur pour ne pas la repasser

que peut-il arriver entre deux soeurs qui s'aiment si fort que le tonnerre ne les atteint pas? conjurer le sort, croire au bon Dieu, laisser le destin s'installer entre le blanc et le noir des négatifs, brûler les étapes et faire sauter le décor, refuser la copie, la reproduction de l'une en l'autre, nier le lien de parenté ou bien soustraire la petite au toucher de la lentille, effacer le carnaval, reprendre le simulacre par l'envers, remettre $l^{\prime}$ enfante dans un écrin de peau de chamois et refermer le couvercle sans dire un mot il faut enfermer les petites filles, leur refuser le droit de grandir ou encore faire descendre les grandes soeurs des chars allégoriques où on entasse les sexes de femmes tableau de seins et de fesses dépareillés grotesque, femmes coupées d'un visage bien à elles

pour que sa petite soeur grandisse en santé, Elvira surveillait les moindres détails de sa croissance physique s'assurait d'une saine alimentation, de visites médicales régulières, d'une hygiène rigoureuse, des meilleurs soins, elle l'éduquait la vêtait de jolies robes propres décentes la présentait aux voisins sous l'apparence de la respectabilité elle campait Julienne avec ses casse-tête ses Lego et ses jeux savants un fruit une boîte de jus derrière la scène sûre de son rôle de soeur/mère

quelque chose échappe toujours aux mères le mouvement le passage de la tendresse à l'indifférence la coloration des joues les pertes le long du chemin les miettes inutiles envolées dans la forêt urbaine le côté sauvage d'une tête décoiffée qui ne retrouve plus les pièces du casse-tête que l'on croit assembler un bras ici une jambe là un oẹil mauve quelques cheveux le derrière d'un homme la queue d'un singe des triangles à peupler des déserts de pyramides et de bouches rouges à la limite du sourire au milieu d'une étendue de noir

Elvira n'avait pas prévu l'échappée de Julienne elle n'avait pas prévu que celle-ci lui grandirait entre les pattes réclamant sa part du butin elle $\mathrm{n}^{\prime}$ avait pas vu venir le coup le photographe avait tout senti d'avance habitué au jeu des soeurs à l'échange difficile des expériences à l'âge à la. beauté de l'enfance n'avait-il pas suggérél'encadrement de la fillette nue dans l'image pour faire vrai pour rire pour s'amuser pour surprendre l'ingénu et l'obscène l'innocence des jeunes et le savoir des grands la douceur et l'amertume c'était presque de l'art entre l'érotisme et la 
pornographie le regard malsain d'une photographie rhabiller la petite pour qu'elle ne prenne pas froid Elvira n'en avait qu'au corps de l'autre elle n'avait pas prévu que la pellicule s'infiltrerait dans la pensée de sa soeur tel un ruisseau dans les veines gonflées de désirs

Julienne offrait le charme la candeur la douceur l'ignorance son corps habitait l'objectif s'enroulait serpentait le lit ouvrait les jambes sans se plaindre faisait comme sa grande soeur qui lui avait tout appris elle remettait du rouge sur ses lèvres roses accrochait des petits coeurs à ses mamelons amorçait un dialogue infini avec ses catins toute nues quand Étienne s'approchait pour jouer avec elle, elle riait, ça chatouille partout

Elvira attendant dans le corridor plantée sur un tabouret ridicule sa peau craquait la pression des ongles laisse une odeur de sang sur la paume des mains dans les coulisses du cerveau de la femme une peine démesurée se glisse ronge l'intérieur gruge le souvenir se demande à quel moment elle a eu tort se penche étouffe les pleurs le cri la chute la plainte assourdie poignée au fond de la gorge tous les pénis avalés en rang comme des soldats face au mur face à la mémoire des filles blanchie à la chaux elle s'était mise au lit pour elle elle avait servi la table pour elle elle avait tout donné mieux qu'une mère pour rien pour en arriver là pour reproduire le modèle jumelles, n'étaient-elles pas presque jumelles filles presque identiques

Deux soeurs rompues deux amours éloignés se parlent parfois une langue semblable les sons brûlent la chair de l'une les mots se fragmentent ne s'entendent plus dorment de moins en moins le sens se faufile Julienne ne vit plus que par impressions le photographe a un livre sur Gauguin qu'elle aime se veut comme les femmes du peintre colorée pleine mûre au coeur d'une végétation délirante délivrée

Elvira vit dans le rêve la vie ne comprend plus le détour la nuance le détail s'acharne à parler comme le père demande exige condamne attend refuse son amour comment vivre sans l'amour d'Elvira, les bras d'Elvira autour du cou, de la taille, le souffle d'Elvira dans le dos la nuit quand il gèle et que même l'asphalte a froid, le sourire d'Elvira quand je me lève, quand elle me voit, la force le silence d'Elvira? Julienne étrangement le pouvait on avait teint les cheveux de la petite $d^{\prime}$ 'un roux criard pour faire plus dramatique, selon Étienne, on lui mettait des robes provocantes accrochantes on l'affichait lui faisait la fête tournait autour d'elle comme des mouches noires, l'adulait, Pretty Baby l'appelait-on, beau bébé

Le photographe s'amusait à recréer le décor de Storeyville en faisait toute une histoire préparait des scénarios loufoques et en remettait ne 
vivait que par l'excès la fascination d'Étienne se jouait entre la lentille, l'oeil et le désir de capturer la beauté

Julienne dorénavant contenue dans cette petite bouche avide, dans la sensualité de cette poitrine encore sage, une petite mer intérieure, une avenue de métropole un dimanche après-midi, un champ vide, une vague sans écume, une plage déserte, un sable si fin que les oiseaux s'y posaient timidement à la fin du jour pour s'envoler aussitôt aucune retenue aucune censure un abandon une confiance un plaisir une jouissance de se savoir regardée de sentir son corps longtemps retenu de bouger de n'appartenir qu'à des doigts qui courent un nid de fourmis, se figurait-elle tous ces picotements cette chaleur poétique Julienne reconnaissait dans tous ses gestes un destin le sien une conviction celle d'être là pour un instant le temps de l'instantané un jour elle ferait du cinéma

Le coeur d'Elvira a éclaté en morceaux. Elle a tué Étienne d'un coup de couteau. La petite s'est enfuie. On l'a revue en train de se promener sur le trottoir au coin de Sainte-Catherine et Papineau. Elvira ne reçoit jamais de visite. Dans sa cellule, elle a collé au mur des photos de sa petite soeur vêtue d'une jolie robe blanche en tricot. 